\title{
HIERARCHICAL CLASSIFICATION OF POLARIMETRIC SAR IMAGE BASED ON STATISTICAL REGION MERGING
}

\author{
F. Lang ${ }^{\text {a*, }}$ J. Yang ${ }^{\text {a }}$ L. Zhao ${ }^{\text {a }}$, D. Li $^{\text {a }}$ \\ ${ }^{a}$ State Key Laboratory of Information Engineering in Surveying, Mapping and Remote Sensing, Wuhan University, 129 Luoyu \\ Road, Wuhan, 430079, China - *langfk@whu.edu.cn
}

Commission VII, Working Group VII/4

KEY WORDS: Polarimetric SAR, Classification, Statistical Region Merging, Segmentation, Region merging, Hierarchical

\begin{abstract}
:
Segmentation and classification of polarimetric SAR (PolSAR) imagery are very important for interpretation of PolSAR data. This paper presents a new object-oriented classification method which is based on Statistical Region Merging (SRM) segmentation algorithm and a two-level hierarchical clustering technique. The proposed method takes full advantage of the polarimetric information contained in the PolSAR data, and takes both effectiveness and efficiency into account according to the characteristic of PolSAR. A modification of over-merging to over-segmentation technique and a post processing of segmentation for SRM is proposed according to the application of classification. And a revised symmetric Wishart distance is derived from the Wishart PDF. Segmentation and classification results of AirSAR L-band PolSAR data over the Flevoland test site is shown to demonstrate the validity of the proposed method.
\end{abstract}

\section{INTRODUCTION}

Polarimetric synthetic aperture radar (PolSAR) provides useful information in a diverse number of applications. Classification of PolSAR imagery has been an important research topic for the last two decades and many supervised and unsupervised classification methods have been proposed. Up till now, the main classification methods are pixel-based which have some limitations: 1) they are noise-sensitive, which leads to piecemeal result map because of the inherent speckle noise of SAR image, 2) they are not convenient for updating Geographic Information System (GIS) database. Thus the object-oriented classification method based on image segmentation technique in Remote Sensing (RS) has been a hot research topic in recent years.

Many segmentation methods of PolSAR data have been developed. A hierarchical stepwise optimization algorithm derived from hierarchical clustering is proposed by Beaulieu and Touzi (Beaulieu and Touzi, 2004). The basic idea of this technique is merging the segments with the minimum criterion value iteratively. Stepwise criterions for one-look and multilook homogeneous and textured scene are derived from maximum-likelihood approach based on Wishart and $\mathrm{K}$ distribution separately. And a new stepwise criterion based on Fisher distribution is proposed by Bombrun and Beaulieu later (Bombrun and Beaulieu, 2008. Bombrun et al. 2009, 2011). Furthermore, a filtering and segmentation algorithm of PolSAR data based on Binary Partition Tree (BPT) is presented by Alonso-Gonzalez et al. (2010, 2011). This method is based on a region-merging and multi-scale present technique that creates the tree by keeping track of the merging steps and presents the image at any level by BPT pruning.

Though the segmentation methods based on hierarchical clustering and region-merging techniques achieve good results, they usually have high time and space complexity, which do bad to large data processing. The Statistical Region Merging (SRM) technology, which is a new image segmentation algorithm belonging to the field of Pattern Analysis and Machine Intelligence (Nock and Nielsen, 2004, 2005), is first introduced to PolSAR classification by $\mathrm{Li}$ et al. (Li et al., 2008). The SRM segmentation algorithm has an optimal time and space complexity. It does not depend on the distribution of the data and has an excellent performance in coping with significant noise corruption, which makes it very competent for segmentation of SAR image which usually has strong speckle noise.

The aim of his paper is to present a new object-oriented classification method which takes both effectiveness and efficiency into account according to the characteristic of PolSAR data. To make sure the efficiency of the algorithm, a hierarchical clustering technique introduced by Bombrun and Beaulieu is adopted in this paper, which directly performs on the initial segmentation result rather than iterative clustering method (Bombrun and Beaulieu, 2008). And a symmetric revised Wishart distance criterion is derived from the Wishart PDF and is used as the classification criterion.

\section{SRM SEGMENTATION}

The Statistical Region Merging (SRM) algorithm is based on a model of image generation that captures the idea of formulating image segmentation as an inference problem (Nock 2001, Nock and Nielsen, 2004), namely, it is the reconstruction of regions on the observed image, based on an unknown theoretical (true) image on which the true regions are statistical regions whose borders are defined from a simple theorem. The SRM algorithm belongs to the family of region growing and merging techniques which usually work with a statistical test to decide the merging of regions. As long as the approach is greedy, two essential components participate in defining a region merging algorithm: the merging predicate which confirms whether the adjacent regions are merged or not and the order followed to test the merging of regions. 


\subsection{Merging Predicate}

From the Nock and Nielsen model the following merging predicate are derived for RGB images (Nock 2004):

$$
P\left(R, R^{\prime}\right)=\left\{\begin{array}{rc}
\text { true. } & \max _{a \in\{R, G, B\}}\left|\overline{R_{a}^{\prime}}-\overline{R_{a}}\right| \leq \sqrt{b^{2}(R)+b^{2}\left(R^{\prime}\right)} \\
\text { false. } & \text { otherwise }
\end{array}\right.
$$

where

$$
b(R)=g \sqrt{\frac{1}{2 Q|R|} \ln \frac{\left|R_{|R|}\right|}{\delta}}
$$

and

$$
\begin{aligned}
& \overline{R_{a}}=\text { the observed average for channel } a \text { in region } R \\
& R^{\prime}=\text { the adjacent region of region } R \\
& \delta=\text { the maximum probability when } P\left(R, R^{\prime}\right)=\text { false, } \\
& \text { which is usually set very small } \\
& Q \text { is a parameter which makes it possible to quantify } \\
& \text { the statistical complexity of the ideal segmentation } \\
& \text { imagery and the statistical hardness of the task as well } \\
& |R|=\text { the pixel number in region } R,|\cdot| \text { stands for } \\
& \text { cardinal } \\
& R_{|R|} \text { stands for the set of regions with n pixels. }
\end{aligned}
$$

\subsection{Merging Order}

For an observed image $I$, there are $N<2|I|$ couples of adjacent pixels in 4-connexity. Let $S_{\mathrm{I}}$ be the set of these couples, and $f(p$, $p^{\prime}$ ) be a real-valued function, with $p$ and $p^{\prime}$ a couple of adjacent pixels in $I$. Instead of stepwise optimization tactics, Nock and Nielsen propose to adopt a pre-ordering strategy. With this strategy, the SRM algorithm can be described as follows: first sort the couples of $\mathrm{S}_{\mathrm{I}}$ in increasing order of $f\left(p, p^{\prime}\right)$, and then traverse this order only once. For any current couple of pixels ( $p$, $\left.p^{\prime}\right) \in \mathrm{S}_{\mathrm{I}}$, if $R(p) \neq R\left(p^{\prime}\right)(R(p)$ stands for the region to which $p$ belongs), make the test $P\left(R(p), R^{\prime}\left(p^{\prime}\right)\right)$, and merge $R$ and $R^{\prime}$ if it returns true.

The simplest sort function $f$ is defined as follows:

$$
f\left(p, p^{\prime}\right)=\max _{a \in\{R, G, B\}}\left|\overline{N_{a}\left(p_{a}\right)}-\overline{N_{a}\left(p_{a}{ }^{\prime}\right)}\right|
$$

where $\overline{N_{a}\left(p_{a}\right)}=$ the observed mean of the region defined by the set of points in channel $a$ that are within Manhattan distance $\leq \Delta$ to $p$, and that are closer to $p$ than to $p$ '.

More sort functions and merging predicates could be defined, which could improve the speed and quality of segmentation.

\subsection{From Over-merging to Over-segmentation}

Nock and Nielsen have proved that with high probability SRM algorithm would get an over-merging result of the ideal segmentation image (Nock and Nielsen, 2004, 2005). But for classification application, the segmentation result must be an over-segmentation of the ideal segmentation. So we should replace the merging predicate (1) by a slightly stricter one.

Remark that provided regions $R$ and $R$ ' are not empty, we have

$$
b\left(R, R^{\prime}\right) \leq \sqrt{b^{2}(R)+b^{2}\left(R^{\prime}\right)}
$$

Where

$$
b\left(R, R^{\prime}\right)=g \sqrt{\frac{1}{2 Q}\left(\frac{1}{|R|}+\frac{1}{\left|R^{\prime}\right|}\right) \ln \frac{2}{\delta}}
$$

So the merging predicate we used is

$$
P\left(R, R^{\prime}\right)=\left\{\begin{array}{rc}
\text { true. } & \max _{a \in\{R, G\}}\left|\overline{R_{a}}{ }^{\prime}-\overline{R_{a}}\right| \leq b\left(R, R^{\prime}\right) \\
\text { false. } & \text { otherwise }
\end{array}\right.
$$

\subsection{Post-Processing of Segmentation}

When using the merging predicate defined by Eq. (6) an oversegmentation result can be obtained easily. But the result usually has many single-pixel noises, which do good to preserving the details and point objects whereas do harm to the following classification processing. So it is demanded to treat these single-pixel noises specially instead of merging them to their background regions directly.

The single-pixel noises which are supposed to be merged should follow three basic principles:

1) The pixel number of the noise regions is measured by an adaptive threshold $N_{\text {th }}$ which is related to the magnitude of image size, look and the statistical complexity $Q$.

2) The number of adjacent regions of the noises is only one, suggesting that the noise is an island inside its adjacent region.

3) The gradient between noises and their adjacent regions is no larger than a threshold $G_{\text {th }}$ in order to preserve strong point objects.

The noise region will be merged ONLY all of the three conditions are satisfied. Otherwise, the noise will be set aside. The proposed empirical formula of $N_{\text {th }}$ is very simple:

$$
N_{t h}=\ln \frac{|I|}{Q}
$$

Where $\quad|I|$ is the total pixel number of image $I$.

$N_{\text {th }}$ is only a function of image pixel number and the statistical complexity $Q$, because the SRM algorithm is independent of look.

\section{HIERARCHICAL CLASSIFICATION}

Hierarchical stepwise optimization clustering is one of the most common methods in segmentation and classification (AlonsoGonzalez et al., 2010, 2011, Beaulieu and Touzi, 2004, 
Bombrun et al., 2008, 2009, 2011). The basic idea of this technique is merging the segments with the minimum criterion value iteratively. Because of the "stepwise optimization" and "multi-level" characteristics, the agglomerative hierarchical clustering algorithm can usually reach a higher accuracy than non-hierarchical clustering algorithm. However, the time complexity will increase progressively when the number of segments is large (Theodoridis and Koutroumbas, 2009). Therefore a two- or three- level hierarchical clustering strategy is badly needed for large dataset.

In this section a new segmentation-based two-level hierarchical clustering algorithm is presented. A revised symmetric Wishart distance $d_{S W}$ is defined as the stepwise criterion, which is a symmetric version of the Wishart distance measure derived from the complex Wishart distribution (Lee et al., 1994).

\subsection{Symmetric Revised Wishart Distance}

The well known Wishart distance which is derived from the complex Wishart distribution based on ML (Maximum Likelihood) classification principle is defined as (Lee et al., 1994)

$$
\begin{aligned}
d_{W}\left(T, V_{m}\right) & =-\frac{1}{n}\left(\ln P_{n, \Sigma}(T)-C\right) \\
& =\ln \left|V_{m}\right|+\operatorname{Tr}\left(V_{m}^{-1} T\right)
\end{aligned}
$$

Where $\quad T$ is a sample coherency matrix $V_{m}$ is the cluster center of the $m$ th class $P_{n, \Sigma}(T)$ is the probability density of $T$

$C$ is an assemblage of variable and constant which is independent of $V_{m}$

$\operatorname{Tr}(\cdot)$ denotes the trace operation.

It is not symmetric while the symmetry character is the most basic demand when measuring the distance between two regions. A possible modification is defined as (Anfisen et al., 2007)

$$
\begin{aligned}
d_{S W}\left(T_{i}, T_{j}\right) & =\frac{1}{2}\left(d_{W}\left(T_{i}, T_{j}\right)+d_{W}\left(T_{j}, T_{i}\right)\right) \\
& =\frac{1}{2}\left[\ln \left|T_{i} \| T_{j}\right|+\operatorname{Tr}\left(T_{i}^{-1} T_{j}+T_{j}^{-1} T_{i}\right)\right]
\end{aligned}
$$

However, the distance is derived from Eq. (8) directly. This may lead to mistakes. As is known that Eq. (8) is a simplification of eliminating $C$ which is independent of $V_{m}$, but is not independent of $T$. The revised version of $d_{W}$ is

$$
\begin{aligned}
d_{R W}\left(T, V_{m}\right) & =-\left(\ln P_{n, \Sigma}(T)-C^{\prime}\right) \\
& =n \ln \left|V_{m}\right|+n T r\left(V_{m}^{-1} T\right)-(n-q) \ln |T|
\end{aligned}
$$

Where $C^{\prime}$ is independent of $T$ as well as $V_{m}$. And its symmetric version is

$$
\begin{aligned}
d_{S R W}\left(T_{i}, T_{j}\right) & =\frac{1}{2}\left(d_{R W}\left(T_{i}, T_{j}\right)+d_{R W}\left(T_{j}, T_{i}\right)\right) \\
& =\frac{1}{2}\left[n \operatorname{Tr}\left(T_{i}^{-1} T_{j}+T_{j}^{-1} T_{i}\right)+q\left(\ln \left|T_{i} \| T_{j}\right|\right)\right]
\end{aligned}
$$

The main difference of Eq. (11) and Eq. (9) is that Eq. (11) is dependent on $n$ and $q$, which can be considered as weight parameters.

\subsection{Hierarchical Classification}

The hierarchical classification method used in this paper can be defined as follows (Bombrun et al., 2008):

1) Suppose there are $N$ regions after SRM over-segmentation processing of the original PolSAR data.

2) First of all, a threshold of pixel number $T$ is confirmed. Then, pick out all the $L$ big regions whose pixel number is larger than $T$ and all the $S$ small regions whose pixel number is smaller than $T$.

3) Calculate the distances among the $L$ big regions according to Eq. (11). Find and merge the two regions with the smallest $d_{S R W}$.

4) Stop, if the big regions reach the desired number $M$; otherwise, go to step 3).

5) Calculate the distances between the $S$ small regions and the $M$ class centres according to Eq. (8). Assign the $S$ small regions into the nearest classes.

With a stepwise optimization strategy, one of the main advantages of this method compared with the commonly used ML classifier is that the classification result of big regions, which occupy the majority of the image, is immune to the initial classification.

\section{EXPERIMENTS AND DISCUSSIONS}

\subsection{Experimental Data}

The AirSAR L-band PolSAR data obtained by NASA JPL over the Flevoland test site, Netherlands is used for the experiments. The size of original data is $1024 \times 750$ pixels and the Pauli-RGB image of the 4-look PolSAR data is shown in Figure 1, from which we can see that this image covers a large agricultural area of flat topography and homogeneous soils. The data has been used by Lee et al. for the pixel-based classification research, and the ground truth map of 11 classes are identified and shown in Figure 2, consisting of eight crop classes from stem beans to wheat, and three other classes of bare soil, water, and forest (Lee et al., 2001).

\subsection{Experiments}

First, the Pauli-RGB image combined with the diagonal elements of coherence $T, 1 / 2|\mathrm{HH}+\mathrm{VV}|^{2}, 1 / 2|\mathrm{HH}-\mathrm{VV}|^{2}, 2|\mathrm{HV}|^{2}$ is used to perform the improved SRM segmentation whose merging predicate is defined by Eq. (6). Figure 4 shows the result when the Manhattan distance $\Delta=2, Q=32$, and $\delta=1 /(6|I|)^{2}$. And the number of segments obtained is 1131 , which is much less compared with the number of total pixels. From the segmentation map we can see that there are nearly no speckle noises and the pixels in homogeneous regions are clustered together correctly. 
As a comparison, the BPT segmentation algorithm was run on the PolSAR data. Figure 7 shows the result when the dissimilarity measure selects the symmetric revised Wishart dissimilarity, and the pruning threshold $\delta_{p}=-0.9 \mathrm{~dB}$. The number of segments obtained is 4863 , which is much more than the number of SRM segments. And some heterogeneous pixels are over-merged together (marked out by ellipse). Other combinations of parameters were tested. The results are not better than the result shown in Figure 7.

After the segmentation, the average coherence matrixes of all the segments are calculated and then the hierarchical classification method defined in section 3.2 is performed. Figure 6 shows the result when the threshold of pixel number is $T=40$ and class number is $M=36$.

Other combinations of parameters were also used. It is found that when $M$ is too small, some different classes of objects will be mixed into the same one.

The result shown in Figure 9 is get when replacing $d_{S R W}$ with $d_{S W}$ while the other parameters remain unchanged in order to perform the validity of the new distance measure.

\subsection{Accuracy assessment}

As is known that the unsupervised classification result does not have meaningful labels initially. To compare with the ground truth map, each category in the unsupervised classification result must be associated with a ground truth label. This can be accomplish by finding a mapping of generic labels of unsupervised classification result to ground truth class names that maximizes the overall accuracy, which is equivalent to finding the mapping that maximizes the trace of the classification confusion matrix $(\mathrm{Yu}, 2011)$. However, this method is not objective because the situation may occur with great possibility: an excess class is labelled to one of the ground truth classes just because only a few pixels or segments are classified into this class mistakenly.

In this paper the mapping is accomplished as follows: compare the unsupervised classification result with the ground truth map and the Pauli-RGB image, and label each category to the class which the majority segments of the category belong to.

After finishing the mapping, the accuracy of the proposed classification method can be measured by the overall accuracy, the individual class accuracies and the Kappa coefficient.

Figure 5 is the merged classification map after mapping Figure 6 onto Figure 2. The confusion matrix is listed in Table 1 . Because the ground truth can not provide a label for each pixel of the entire image, the accuracy calculation is limited to only those pixels where the ground truth map covers.

Similarly, Figure 8 is the merged classification map after mapping Figure 9 onto Figure 2. The confusion matrix is listed in Table 2.

From Table 1 and Table 2 we can see that the total accuracy of the classification result using $d_{S R W}$ can reach $91.25 \%$, and the Kappa coefficient is 0.901135 . While the total accuracy of the classification using $d_{S W}$ is only $80.92 \%$, and the Kappa coefficient is 0.78641 . However, we notice that the accuracy of Beet is just $40.49 \%$, which is far lower than the total accuracy. By comparing Figure 2 with Figure 6, we can find that some segments of the Beet class (marked out by ellipse) are mixed with some other classes (marked out by rectangle) which are not included in the ground truth map. If these segments are labelled as Beet according to the method of maximizes the overall accuracy, the accuracy of Beet can reach up to $78.62 \%$. This is not really objective.

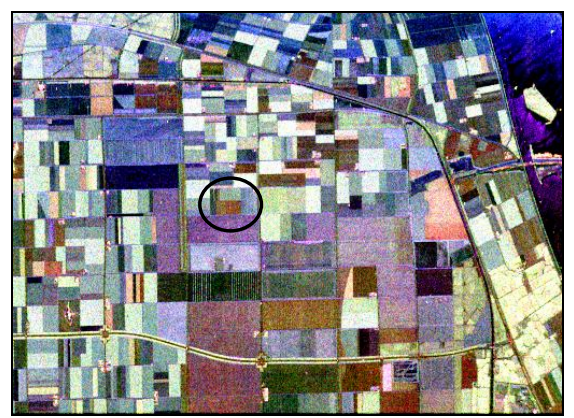

Figure 1. Pauli-RGB image of PolSAR data

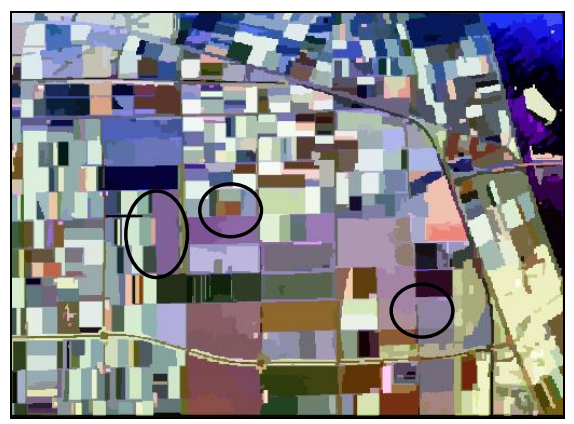

Figure 4. SRM segmentation map

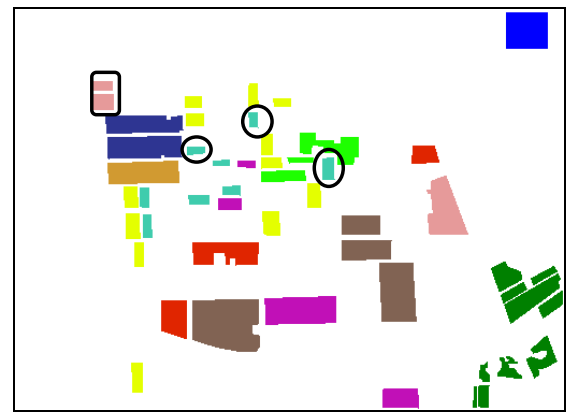

Figure 2. Ground truth map

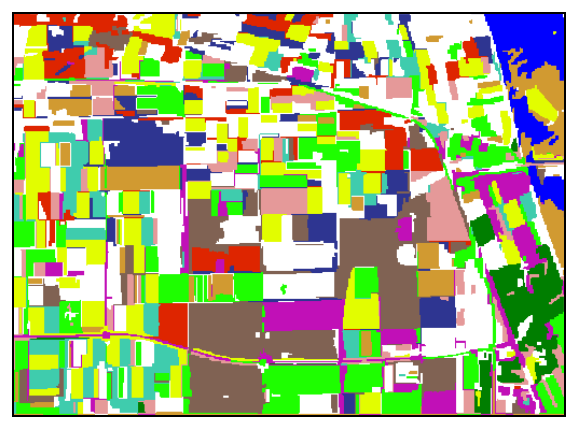

Figure 5. Merged classification map of Figure 6

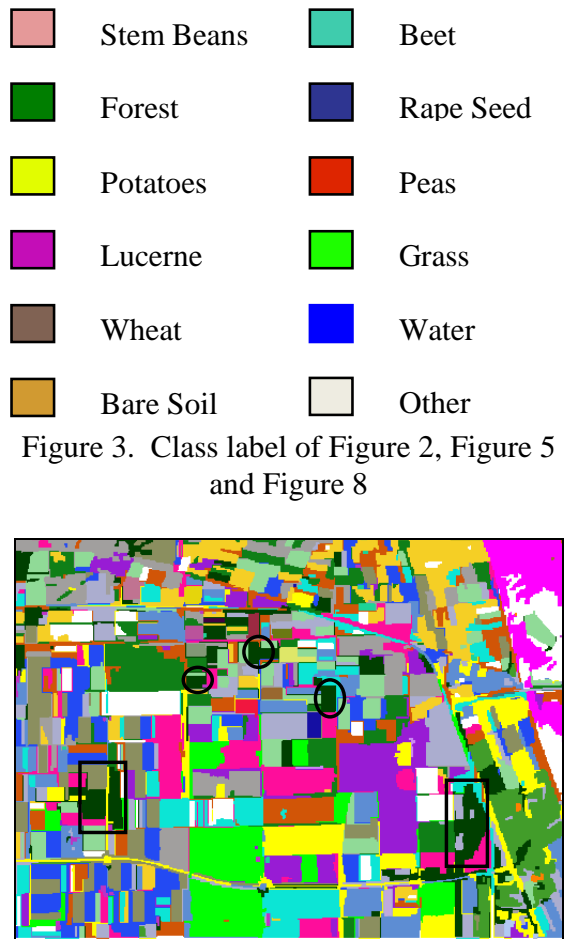

Figure 6. Classification map using $d_{S R W}$ 


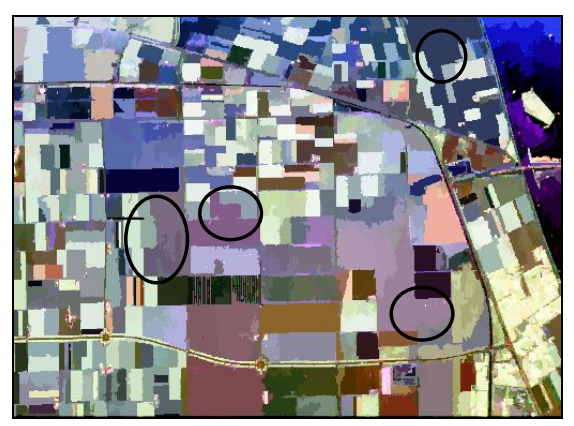

Figure 7. BPT segmentation map

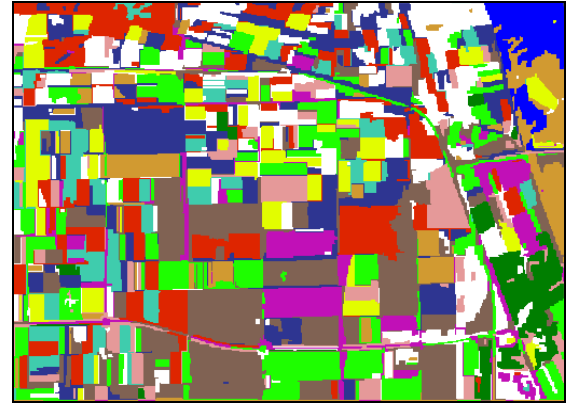

Figure 8. Merged classification map of

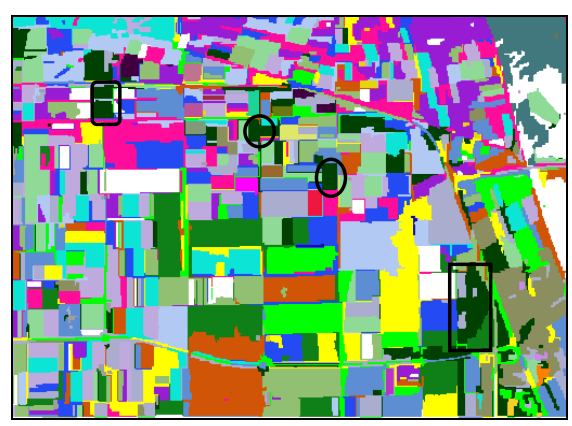

Figure 9. Classification map using $d_{S W}$ Figure 9

Table 1. Confusion matrix of the classification result using $d_{S R W}$

\begin{tabular}{|c|c|c|c|c|c|c|c|c|c|c|c|c|c|}
\hline & $\begin{array}{l}\text { Stem } \\
\text { beans }\end{array}$ & Forest & $\begin{array}{c}\text { Potatoe } \\
\text { s }\end{array}$ & $\begin{array}{c}\text { Lucern } \\
\mathrm{e}\end{array}$ & Wheat & $\begin{array}{c}\text { Bare } \\
\text { soil }\end{array}$ & Beet & $\begin{array}{l}\text { Rape } \\
\text { seed }\end{array}$ & Peas & Grass & Water & Total & user_acc \\
\hline Stem beans & 1832 & 28 & 0 & 0 & 0 & 0 & 88 & 0 & 0 & 0 & 0 & 1948 & $94.05 \%$ \\
\hline Forest & 0 & 2995 & 0 & 0 & 0 & 0 & 0 & 0 & 0 & 0 & 0 & 2995 & $100.00 \%$ \\
\hline Potatoes & 0 & 119 & 2840 & 0 & 0 & 0 & $\mathbf{0}$ & 0 & 0 & 0 & 0 & 2959 & $95.98 \%$ \\
\hline Lucerne & 0 & 0 & 0 & 1754 & 0 & 0 & $\mathbf{0}$ & 0 & 0 & 0 & 0 & 1754 & $100.00 \%$ \\
\hline Wheat & 0 & 0 & 0 & 0 & 6029 & 0 & 175 & 17 & 89 & 0 & 0 & 6310 & $95.55 \%$ \\
\hline Bare soil & 0 & 0 & 0 & 0 & 0 & 1340 & $\mathbf{0}$ & 0 & 0 & 0 & 51 & 1391 & $96.33 \%$ \\
\hline Beet & 0 & 0 & 9 & 0 & 0 & 0 & 498 & 0 & 0 & 0 & 0 & 507 & $98.22 \%$ \\
\hline Rape seed & 0 & 0 & 0 & 0 & 0 & 0 & $\mathbf{0}$ & 2224 & 0 & 0 & 0 & 2224 & $100.00 \%$ \\
\hline Peas & 0 & 0 & 1 & 0 & 0 & 0 & 0 & 0 & 2214 & 0 & 0 & 2215 & $99.95 \%$ \\
\hline Grass & 0 & 0 & 0 & 864 & 0 & 0 & O & 0 & 0 & 1451 & 0 & 2315 & $62.68 \%$ \\
\hline Water & 0 & 0 & 0 & 0 & 0 & 0 & 0 & 0 & 0 & 0 & 1275 & 1275 & $100.00 \%$ \\
\hline Other & 17 & 111 & 0 & 0 & 0 & 0 & 469 & 289 & 0 & 17 & 0 & 903 & \\
\hline Total & 1849 & 3253 & 2850 & 2618 & 6029 & 1340 & 1230 & 2530 & 2303 & 1468 & 1326 & 26796 & \\
\hline prod_acc & $\begin{array}{c}99.08 \\
\%\end{array}$ & $\begin{array}{c}92.07 \\
\%\end{array}$ & $99.65 \%$ & $67.00 \%$ & $\begin{array}{c}100.00 \\
\%\end{array}$ & $\begin{array}{c}100.00 \\
\%\end{array}$ & $40.49 \%$ & $87.91 \%$ & $\begin{array}{c}96.14 \\
\%\end{array}$ & $\begin{array}{c}98.84 \\
\%\end{array}$ & $\begin{array}{c}96.15 \\
\% \\
\end{array}$ & & \\
\hline \multicolumn{14}{|c|}{ Total accuracy $=24452 / 26796=91.25 \% \quad$ Kappa $=0.901135$} \\
\hline
\end{tabular}

Table 2. Confusion matrix of the classification result using $d_{S W}$

\begin{tabular}{|c|c|c|c|c|c|c|c|c|c|c|c|c|c|}
\hline & $\begin{array}{l}\text { Stem } \\
\text { beans }\end{array}$ & Forest & Potatoes & Lucerne & Wheat & Bare soil & Beet & $\begin{array}{l}\text { Rape } \\
\text { seed }\end{array}$ & Peas & Grass & Water & Total & user_acc \\
\hline Stem beans & 1378 & 28 & 280 & 0 & 0 & 0 & 88 & 0 & 0 & 0 & 0 & 1774 & $77.68 \%$ \\
\hline Forest & 0 & 2995 & 0 & 0 & 0 & 0 & 0 & 0 & 0 & 0 & 0 & 2995 & $100.00 \%$ \\
\hline Potatoes & 0 & 90 & 1772 & 0 & 0 & 0 & 0 & 0 & 0 & 0 & 0 & 1862 & $95.17 \%$ \\
\hline Lucerne & 0 & 0 & 0 & 1754 & 0 & 0 & 0 & 0 & 0 & 0 & 0 & 1754 & $100.00 \%$ \\
\hline Wheat & 0 & 0 & 0 & 0 & 4484 & 0 & 0 & 0 & 89 & 278 & 0 & 4851 & $92.43 \%$ \\
\hline Bare soil & 0 & 0 & 0 & 0 & 0 & 1340 & 0 & 0 & 0 & 0 & 51 & 1391 & $96.33 \%$ \\
\hline Beet & 0 & 0 & 9 & 0 & 0 & 0 & 796 & 0 & 0 & 0 & 0 & 805 & $98.88 \%$ \\
\hline Rape seed & 0 & 0 & 0 & 0 & 0 & 0 & 0 & 2503 & 0 & 17 & 0 & 2520 & $99.33 \%$ \\
\hline Peas & 17 & 29 & 789 & 0 & 1545 & 0 & 0 & 17 & 2214 & 0 & 0 & 4611 & $48.02 \%$ \\
\hline Grass & 0 & 0 & 0 & 864 & 0 & 0 & 0 & 0 & 0 & 1173 & 0 & 2037 & $57.58 \%$ \\
\hline Water & 0 & 0 & 0 & 0 & 0 & 0 & 0 & 0 & 0 & 0 & 1275 & 1275 & $100.00 \%$ \\
\hline Other & 454 & 111 & 0 & 0 & 0 & 0 & 346 & 10 & 0 & 0 & 0 & 921 & \\
\hline Total & 1849 & 3253 & 2850 & 2618 & 6029 & 1340 & 1230 & 2530 & 2303 & 1468 & 1326 & 26796 & \\
\hline prod_acc & $\begin{array}{c}74.53 \\
\% \\
\end{array}$ & $\begin{array}{c}92.07 \\
\%\end{array}$ & $62.18 \%$ & $67.00 \%$ & $\begin{array}{c}74.37 \\
\% \\
\end{array}$ & $\begin{array}{c}100.00 \\
\% \\
\end{array}$ & $64.72 \%$ & $98.93 \%$ & $\begin{array}{c}96.14 \\
\% \\
\end{array}$ & $\begin{array}{c}79.90 \\
\% \\
\end{array}$ & $\begin{array}{c}96.15 \\
\%\end{array}$ & & \\
\hline \multicolumn{14}{|c|}{ Total accuracy $=21684 / 26796=80.92 \%$} \\
\hline
\end{tabular}




\section{CONCLUSIONS}

This paper has presented an unsupervised hierarchical objectoriented classification method of PolSAR image, which is mainly supported by the SRM segmentation technique and hierarchical clustering technique. The SRM algorithm is originally an optical image segmentation method. However, it is demonstrated that the technique can also be used for PolSAR image segmentation by some improvements, which are discussed in section 2.3 and 2.4. A symmetric revised distance measure based on Wishart distribution is derived. And further more, a two-level hierarchical classification based on this distance measure is defined.

Segmentation and classification results of AirSAR L-band PolSAR data over the Flevoland test site are presented. And the quality of the proposed method is assessed by the overall accuracy, the individual class accuracies and the Kappa coefficient. The results indicate that the proposed method, by integrating the advantages of SRM and hierarchical techniques, can reach high classification accuracy, and is an efficient objectoriented classification method for PolSAR image.

\section{REFERENCES}

Alonso-Gonzalez, A., López-Martínez, C., and Salembier, P., 2010. Filtering and segmentation of polarimetric SAR images with binary partition trees. IGARSS 2010, pp. 4043-4046.

Alonso-González, A., López-Martínez, C., and Salembier, P., 2011. Filtering and Segmentation of Polarimetric SAR Data Based on Binary Partition Trees. IEEE Transactions on Geoscience and Remote Sensing, pp. 1-13.

Anfinsen, S. N., Jenssen, R., and Eltoft, T., 2007. Spectral clustering of polarimetric SAR data with Wishart-derived distance measures, in PolInSAR 2007.

http://earth.esa.int/workshops/polinsar2007/papers/140_anfinse n.pdf

Beaulieu, J.-M. and Touzi, R., 2004. Segmentation of textured polarimetric SAR scenes by likelihood approximation. IEEE Transactions on Geoscience and Remote Sensing, 42(10), pp. 2063-2072.

Bombrun, L. and Beaulieu, J.-M., 2008. Segmentation of polarimetric SAR data based on the Fisher distribution for texture modelling. IGARSS 2008, pp. 1-4.

Bombrun, L., Beaulieu, J.-M., Vasile, G., Ovarlez, J.-P., Pascal, F., and Gay, M., 2009. Hierarchical segmentation of Polarimetric SAR images using heterogeneous clutter models, IGARSS 2009, pp. III-5-III-8.
Bombrun, L., Beaulieu, J.-M., Vasile, G., Ovarlez, J.-P., Pascal, F., and Gay, M., 2011. Hierarchical segmentation of Polarimetric SAR images using heterogeneous clutter models, IEEE Transactions on Geoscience and Remote Sensing, 49(2), pp. 726-737.

Lee, J. S., Grunes, M. R., and Kwok, R., 1994. Classification of multi-look polarimetric SAR imagery based on complex Wishart distribution, Int. J. Remote Sensing, 15(11), pp. 229231.

Lee, J. S., Grunes, M. R., Pottier, E., 2001. Quantitative Comparison of Classification Capability: Fully Polarimetric Versus Dual and Single-Polarization SAR. IEEE Transactions on Geoscience and Remote Sensing, 39(11), pp. 2343-2351

Li, H. T., Gu, H.Y., Han, Y. S., Yang, J. H., 2008. Objectoriented Classification of Polarimetric SAR Imagery based on Statistical Region Merging and Support Vector Machine. International Workshop on Earth Observation and Remote Sensing Applications, pp. 1-6.

Nock R., 2001. Fast and reliable color region merging inspired by decision tree pruning, Proceedings of the 2001 IEEE Computer Society Conference on Computer Vision and Pattern Recognition. CVPR 2001, pp. I-271-I-276.

Nock, R. and Nielsen, F., 2004. Statistical region merging, IEEE transactions on pattern analysis and machine intelligence, 26(11), pp. 1452-1458.

Nock, R. and Nielsen, F., 2005. Semi-supervised statistical region refinement for color image segmentation, Pattern Recognition, 38(6), pp. 835-846.

Theodoridis S. and Koutroumbas K., 2009. Pattern Recognition, 4th ed. Elsevier.

Yu P., Qin A. K., Clausi D. A., and Member S., 2011. Unsupervised Polarimetric SAR Image Segmentation and Classification Using Region Growing With Edge Penalty, IEEE Transactions on Geoscience and Remote Sensing, (99), pp. 116.

\section{ACKNOWLEDGEMENTS}

This work is supported by the National Natural Science Foundation of China (60890074), the National 863 Hi-tech R\&D Program of China (2011AA120404) and the Fundamental Research Funds for the Central Universities (201161902020003).

The authors would like to thank the PolSARpro project distributed by ESA to provide the experimental data. 